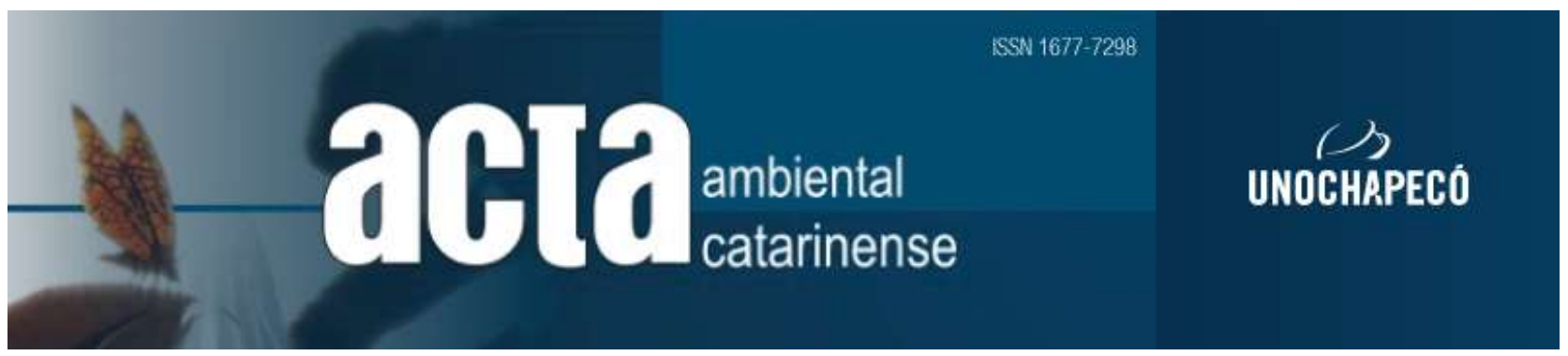

\title{
VULNERABILIDADE DOS SOLOS À EROSÃO HÍDRICA NA SUB-BACIA do Ribeirão do CiPó - PoÇos de Caldas / MG
}

\author{
Adriano Mota Ferreira ${ }^{1}$ \\ Antônio Marciano da Silva ${ }^{2}$ \\ Paulo Henrique Bretanha Junker Menezes ${ }^{3}$ \\ César Henrique Valentino ${ }^{4}$ \\ Cláudio André dos Passos ${ }^{5}$ \\ Maurício Dias Purcino ${ }^{6}$
}

\begin{abstract}
Resumo
A erosão hídrica do solo, ocasionada pela chuva e respectivo escoamento superficial, é a forma mais significativa de sua degradação. Neste trabalho objetivou-se estimar o potencial de perda anual média de solos e sua distribuição espacial na Sub-bacia Hidrográfica do Ribeirão do Cipó (SBRC), e a identificação das áreas de maior vulnerabilidade à erosão hídrica. Assim, foi aplicado o modelo RUSLE (Revised Universal Soil Loss Equation - Renard et al., 1997) implementado em ambiente SIG, com a elaboração e a integração dos mapas dos fatores do modelo. Constatou-se que 53\% da área da SBRC está na classe de moderada vulnerabilidade $18 \%$, na classe de vulnerabilidade muito alta, com base na classificação proposta por Beskow et al. (2009). O uso do solo com mata nativa e reflorestamento concorreram positivamente para a redução da vulnerabilidade à erosão hídrica e, por outro lado, os cultivos anuais e as áreas de solo descoberto e minerado, sem práticas conservacionistas, elevaram significativamente a vulnerabilidade e consequentemente a perda de solo potencial média estimada de 45,14 ( $\mathrm{t} \mathrm{ha}^{-1}$ ano $^{-1}$ ) para a SBRC, situando-a na classe de Muito Alta Vulnerabilidade à erosividade das chuvas.
\end{abstract}

\footnotetext{
${ }^{1}$ Instituto de Ciência e Tecnologia - Programa de Pós-Graduação em Ciência e Engenharia Ambiental, Universidade Federal de Alfenas (UNIFAL - MG).

${ }^{2}$ Professor do Programa de Pós-Graduação em Ciência e Engenharia Ambiental, do Instituto de Ciência e Tecnologia/Universidade Federal de Alfenas (UNIFAL - MG). Professor Emérito da UFLA.

${ }^{3}$ Professor Dr. no Instituto de Ciência e Tecnologia, Universidade Federal de Alfenas (UNIFAL - MG).

${ }^{4}$ Instituto de Ciência e Tecnologia - Programa de Pós-Graduação em Ciência e Engenharia Ambiental, Universidade Federal de Alfenas (UNIFAL - MG).

${ }^{5}$ Instituto de Ciência e Tecnologia - Programa de Pós-Graduação em Ciência e Engenharia Ambiental, Universidade Federal de Alfenas (UNIFAL - MG).

${ }^{6}$ Instituto de Ciência e Tecnologia - Programa de Pós-Graduação em Ciência e Engenharia Ambiental, Universidade Federal de Alfenas (UNIFAL - MG).
} 
Palavras-chave: Geoprocessamento. RUSLE. Erosão hídrica. Solos.

\begin{abstract}
Water erosion is the most significant form of erosion, caused by rainfall and its respective surface runoff. This paper aims to estimate the spatial distribution of the average annual soil loss potential in the Ribeirão do Cipó Hidrographic Basin (SBRC), with the identification of areas with the highest susceptibility to water erosion. The RUSLE (Revised Universal Soil Loss Equation - Renard et al., 1997) model implemented in GIS was applied through the elaboration of the equation factors for later map algebra. It was found that 53\% of the SBRC area is in the class of moderate vulnerability, being also, $18 \%$ in the very high vulnerability class, classification Beskow et al. (2009). Land use with native forest and reforestation contributed positively to reducing vulnerability to water erosion, and, on the other hand, annual crops and areas of uncovered and mined soil, without conservation practices, significantly increased the vulnerability and consequently the loss of the estimated average soil of 45.14 (t. ha ${ }^{-1}$ year $^{-1}$ ) for the SBRC, placing in the Very High Vulnerability to rainfall erosivity class.
\end{abstract}

Keywords: Geoprocessing. RUSLE. Water Erosion. Soils.

\section{INTRODUÇÃo}

A erosão do solo e a degradação de terras agricultáveis consiste em um dos problemas mais iminentes da humanidade, responsáveis pelo decrescimento da qualidade e produtividade do solo, pelo esgotamento das áreas agricultáveis, redução da infiltração, aumento do escoamento superficial e pela deterioração da qualidade da água (SILVA et al., 2007; RODRIGUES et al, 2017).

Avaliações quantitativas da magnitude dos problemas de erosão e as possíveis estratégias de manejo em uma base regional são necessárias para agências de planejamento e desenvolvedores de políticas ligadas ao desenvolvimento sustentável. De acordo com Oliveira et al. (2014), o mapeamento e avaliação da vulnerabilidade do solo à erosão é uma importante ferramenta para o planejamento e gestão dos recursos naturais.

Atualmente, para análises ambientais, os Sistemas de Informação Geográfica (SIG) são ferramentas eficientes devido a sua grande versatilidade na gestão e análise de informações espaciais, contribuindo para avaliações de degradação e planejamento e gestão da terra (EFTHIMIOU et al., 2016; CUIABANO et al., 2017). Sendo assim, análises espaciais de indicadores associados à erosão permitem a identificação de áreas com maior propensão à erosão e transporte de sedimentos, e também, subsídio na recomendação de práticas de conservação mais eficazes para as condições de vulnerabilidade de cada trecho ou setor de uma bacia hidrográfica. De acordo com Gianinetto et al. (2019) a taxa média anual de perda de solo excede a taxa média anual de sua formação, fazendo com que o recurso possa ter comportamento de recurso não renovável.

O município de Poços de Caldas-MG é um dos dois únicos no Brasil que possui autossuficiência no fornecimento de energia elétrica e no abastecimento de água e tratamento do esgoto, propiciadas por concessionárias municipais de serviços públicos, respectivamente Departamento Municipal de Eletricidade - DME, e Departamento Municipal de Água e Esgoto - DMAE. Além disso, o Ribeirão do Cipó com seu reservatório, é responsável por $47 \%$ do abastecimento de água do município (DMAE, 2013).

Devido a esta conjuntura, as ações de pesquisa estão voltadas para a Sub-bacia Hidrográfica do Ribeirão do Cipó (SBRC), que 
assume relevância, pelo uso de seus mananciais, para duas atividades das mais relevantes no contexto dos recursos hídricos: abastecimento público e geração de energia, as quais têm, na presença de sedimentos suspensos, um grande fator de redução de sua eficiência e aumento de custos operacionais.

O modelo RUSLE (Revised Universal Soil Loss Equation) proposto por Renard et al. (1997), consta de uma versão revisada do modelo USLE (Universal Soil Loss Equation), é considerado um modelo simples de predição que permite estimar a erosão hídrica potencial com base na interação entre os fatores erosividade da chuva, erodibilidade do solo, influências do relevo, da cobertura e do manejo do solo, a partir da utilização de ferramentas SIG, permitindo assim, o tratamento das informações de forma espacializada. Inúmeras são as utilizações da RUSLE, assim como de seus fatores. Mello et al. (2015) explicam que métodos utilizados para prever o fator de erosividade das chuvas (fator R) por exemplo, têm sido úteis para o planejamento do uso de terras agrícolas, o que é extremamente importante em termos de mapeamentos regionais associados à erosão.

Buscou-se então neste trabalho, avaliar a vulnerabilidade à erosão hídrica dos solos na Sub-bacia hidrográfica do Ribeirão do Cipó - Poços de Caldas - MG, com o emprego do modelo RUSLE (RENARD et al., 1997) em ambiente SIG.

\section{MATERIAL E MÉtodos}

\subsection{Caracterização da área de estudo}

A Sub-bacia hidrográfica do Ribeirão do Cipó (SBRC), com área de drenagem de $77,8 \mathrm{~km}^{2}$, localiza-se na zona rural do município de Poços de Caldas entre as coordenadas $3325000 \mathrm{~W}$ e $7575000 \mathrm{~S}$ sistema SIRGAS 2000 - Fuso 23K, sendo uma das principais bacias afluentes do Ribeirão das Antas. A bacia faz parte da GD-6, unidade gerencial do comitê de bacias hidrográficas dos afluentes mineiros dos Rios Mogi-Guaçu e Pardo, que são afluentes do Rio Grande, o qual forma posteriormente o Rio Paraná. Na Figura 1 é mostrada a localização da Sub-bacia do Ribeirão do Cipó.

O índice pluviométrico anual na região situa-se entre 1.300 a $1.700 \mathrm{~mm}$ e, segundo a classificação de Köppen, apresentam-se duas variações do clima mesotérmico $(\mathrm{C})$, o Cwa e o Cwb. Na maior parte da área do planalto predomina o Cwb e o Cwa apresenta-se na porção oeste, nos domínios do município de Águas da Prata (MORAES, 2007). A altimetria na SBRC varia entre $1.248,44$ e $1.493,83 \mathrm{~m}$ como mostrado na Figura 2 (a), e na Figura 2 (b) o mapa de declividades da área de estudo, elaborado com base na classificação de EMBRAPA (2006).

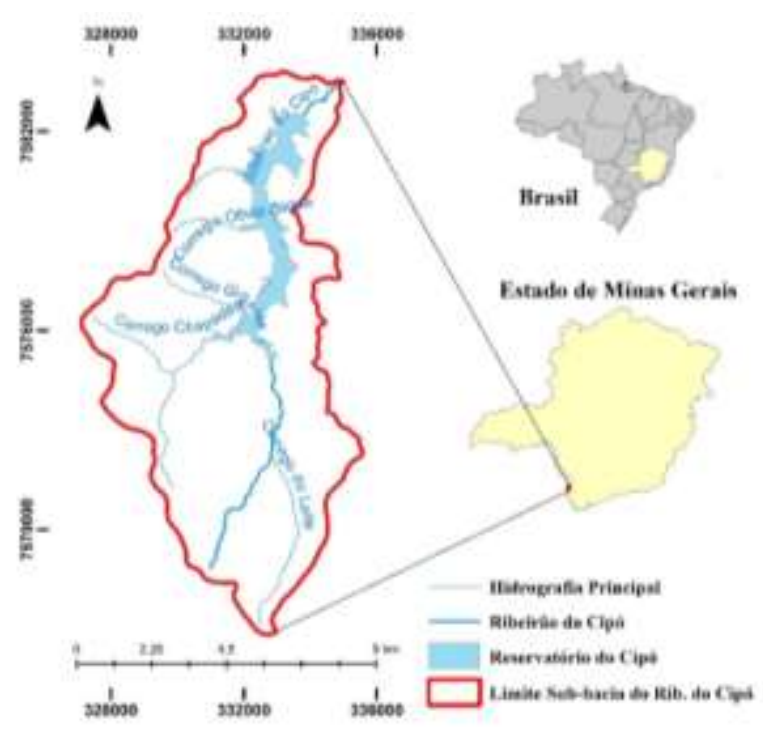

Figura 1 - Localização da Sub-bacia Hidrográfica do Ribeirão do Cipó (SBRC). 
$\mathrm{Na}$ Sub-bacia em estudo, foram mapeadas as classes de uso e cobertura do solo por meio da vetorização de imagem de alta resolução com interpretação visual, baseada no conhecimento das classes de acordo com a caracterização proposta por Araújo Filho et al. (2007). As classes mapeadas enquadram-se nas unidades - Campo associado a umidade, Campo de Altitude, Massas d'água, Cultura, Fragmento de Mata Nativa, Mata Ripária, Pastagem, Reflorestamento e Solo exposto.
Tomando como base o levantamento pedoestratigráfico de Moraes (2007) na região de estudo, as colaborações pessoais de Curi e Silva $^{7}$ e o Mapa de Solos de Minas Gerais (UFV et al., 2010), foram obtidas as classes de solos identificadas na área, assim como suas áreas de ocorrência. Além dos dados associados aos solos, cobertura e manejo, a aquisição e processamentos dos demais dados utilizados serão mostrados adiante.
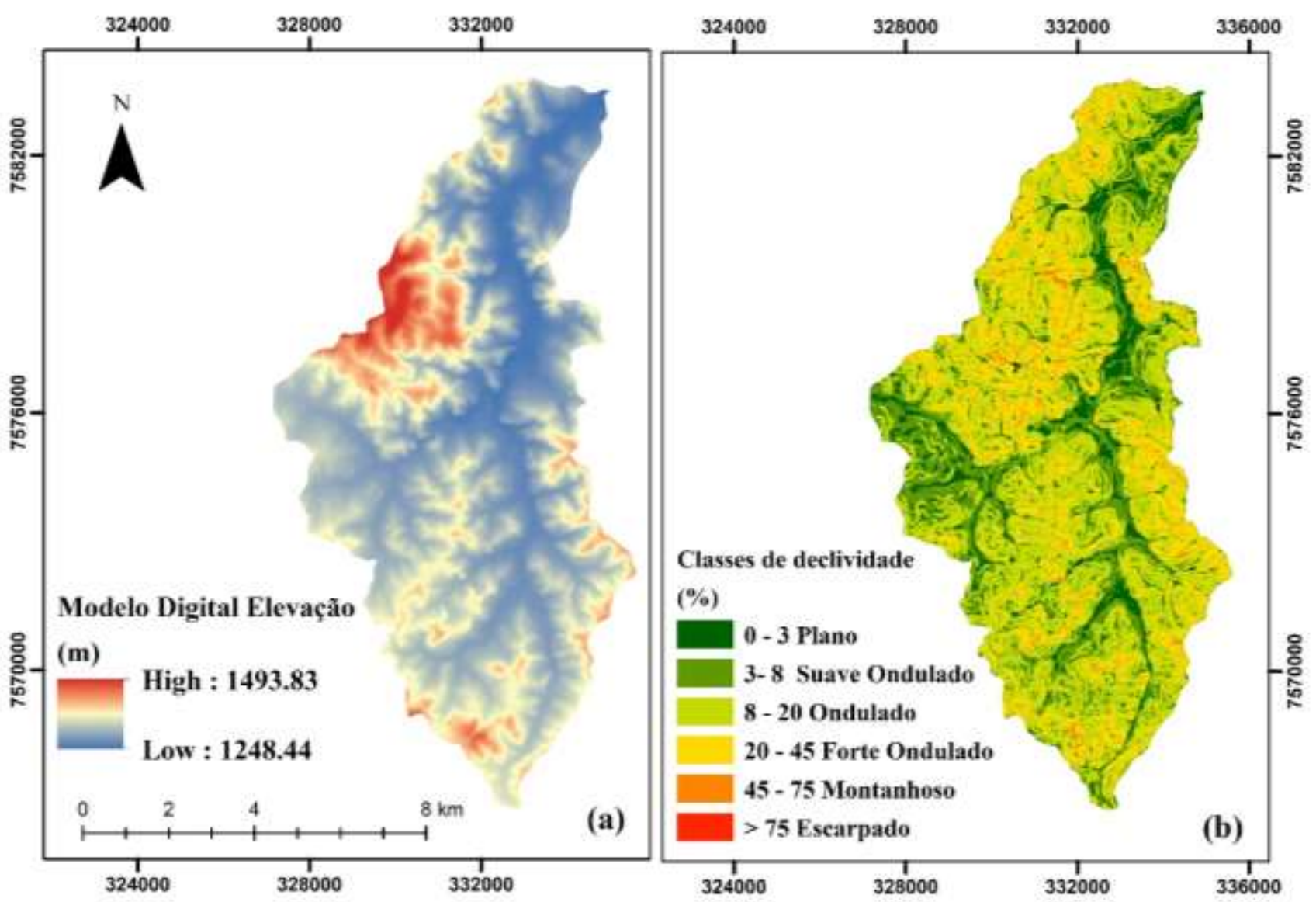

Figura 2 - Modelo digital de elevação (a) e intervalos de classes de declividade (*) (b), na Sub-bacia hidrográfica do Ribeirão do Cipó. (*) Intervalos de acordo com EMBRAPA (2006). Fonte: Elaborado pelo autor.

\footnotetext{
${ }^{7}$ Colaboração dos Professores Dr. Nilton Curi, Dr. Sérgio Henrique Godinho da Silva; - Departamento de Ciência do Solo DCS - Universidade Federal de Lavras - UFLA.
} 


\subsection{Equação Universal de Perda de Solo Re- visada (RUSLE)}

A Equação Universal de Perda de Solo Revisada (Revised Universal Soil Loss Equation - RUSLE) é um modelo paramétrico que foi testado e validado em diversas condições de solo, clima e manejo (RENARD et al., 1997). A RUSLE, implementada em ambiente SIG (Sistema de Informação Geográfica) foi utilizada para estimar a perda média anual de solo na Sub-bacia do Ribeirão do Cipó. A equação é baseada numa função de cinco principais fatores que estão associados à erosão do solo (Equação I), os quais são gerados um a um (separadamente) na forma de mapas convertidos para formato raster, em uma resolução espacial de 30 metros, sendo então combinados a partir da aplicação de álgebra de mapas.

$$
A=R * K * L S * C * P
$$

Onde, A é a taxa de perda média anual de solo por unidade de área $\left(\mathrm{t} \mathrm{ha}^{-1} \mathrm{ano}^{-1}\right)$; $\mathrm{R}$ é o fator de erosividade da chuva (MJ mm ha ${ }^{-1} \mathrm{~h}^{-1}$ ano $^{-}$ ${ }^{1}$ ); K é o fator de erodibilidade do solo ( $\mathrm{t} \mathrm{h} \mathrm{MJ}^{-}$ ${ }^{1} \mathrm{~mm}^{-1}$ ); LS é o fator topográfico (adimensional); C é o fator de cobertura e manejo do solo (adimensional); e P é o fator de práticas conservacionistas (adimensional).

\subsection{Fator de Erosividade da Chuva (R)}

O fator erosividade da chuva (R) representa o potencial natural da chuva em provocar erosão do solo, cuja definição física consiste do produto da energia cinética da chuva pela intensidade média máxima em 30 minutos consecutivos (WISCHMEIER e SMITH, 1978). Neste estudo, para estimativa da erosividade média anual de forma espacialmente distribuída, foi utilizado um modelo geográfico e estatístico desenvolvido por Mello et al. (2013) calculando-se então o fator R para a SBRC em função da latitude, longitude e altitude conforme a Equação (II):

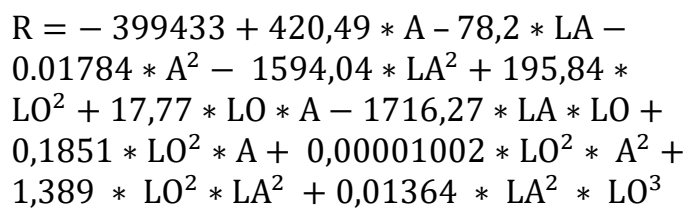

Onde, R é o fator de erosividade da chuva (MJ $\left.\mathrm{mm} \mathrm{ha}^{-1} \mathrm{~h}^{-1} \mathrm{ano}^{-1}\right)$, A é a altitude (m); LA é a latitude e LO é a longitude, ambos em graus decimais negativos. A Figura 3 ilustra a malha regular de pontos, utilizada para a espacialização do fator (R) via Krigagem simples.

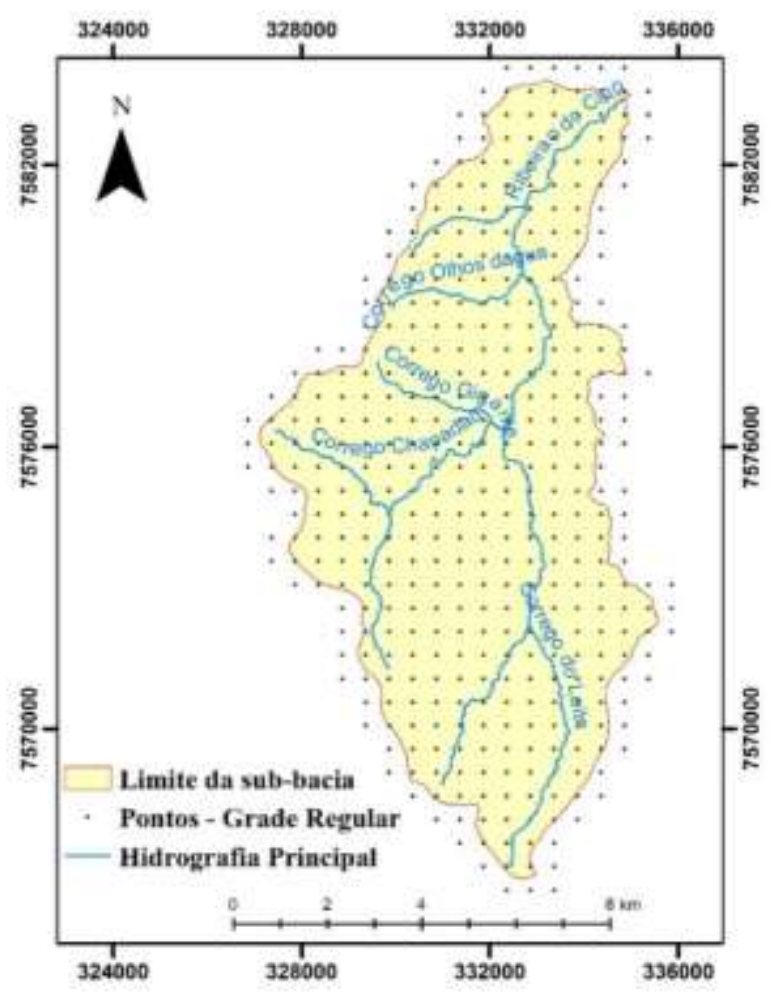

Figura 3 - Malha regular de pontos (500 x 500) metros, utilizada no processamento da erosividade das chuvas (R). Fonte: Elaborado pelo autor.

\subsection{Fator de erodibilidade do solo (K)}

De acordo com Prasannakumar et al. (2012), diferentes tipos de solos são naturalmente mais resistentes ou suscetíveis a erosão do que outros. $\mathrm{O}$ fator $\mathrm{K}$ diz respeito a erodibilidade dos solos, sendo então a susceptibili- 
dade intrínseca de cada solo à erosão, em função de suas características químicas, físicas e pedológicas, como textura, permeabilidade, matéria orgânica, material parental, entre outros (PRADHAN et al., 2012; PRASANNAKUMAR et al., 2012). Os valores utilizados para o processamento do modelo na estimativa das perdas de solo estão descritos na Tabela 1.

Tabela 1 - Classes de solo, áreas e valores utilizados para erodibilidade (K). CX - Cambissolo Háplico; LVA - Latossolo VermelhoAmarelo; RL - Neossolo Litólico; SIV - Solos Indiscriminados de Várzea. Fonte: Elaborado pelo autor.

\begin{tabular}{ccccc}
\hline Solo & Área $\left(\mathrm{km}^{2}\right)$ & $(\%)$ & Fator $\mathrm{K}$ & Referência \\
\hline CX & 16,03 & 22,2 & 0,0355 & Silva et al., (2009) \\
LVA & 42,88 & 59 & 0,01 & Silva et al., (2000) \\
RL & 0,2138 & 0,29 & 0,057 & Oliveira et al., 2014 \\
SIV & 18,58 & 18,5 & 0,042 & Silva (2004) \\
Total & 77,7 & 100 & - & - \\
\hline
\end{tabular}

\subsection{Fator topográfico (LS)}

O fator topográfico (LS) representa a relação entre as perdas de solo em uma área com comprimento de rampa e declividade quaisquer e as perdas de solo correspondentes em uma parcela padrão, caracterizada por 22,13 metros de comprimento com 9\% de declive (WISCHMEIER e SMITH, 1978). Quanto maior a encosta e declive, maiores as perdas de solo (RODRIGUES et al., 2017), ou seja, a inclinação e o comprimento da encosta afetam consideravelmente a taxa de erosão hídrica (GASHAW et al., 2017). No presente trabalho o fator LS foi calculado por meio da metodologia proposta por $\mathrm{McCool}$ et al. (1989) com execução na ferramenta $L S$ TOOL $^{\circledR}$ proposta por Zhang et al., (2013) assim como pós processamento em ambiente SIG.

\subsection{Fator de cobertura e manejo do solo (C) e Fator práticas conservacionistas $(\mathbf{P})$}

$\mathrm{O}$ fator $(\mathrm{C})$ varia de 0 a 1 (adimensional), onde valores próximos de 1 representam áreas com cobertura vegetal quase nula, e, portanto, mais vulneráveis à erosão hídrica. As classes mapeadas, suas ocorrências e seus índices referenciados constam na Tabela 2. O fator $(\mathrm{P})$ de práticas conservacionistas tem por base a maneira pela qual a cultura foi conduzida na área. Corresponde à relação entre as perdas de solo de uma área cultivada com determinada prática e às perdas, quando se planta morro abaixo, mantendo-se as condições padrão de relevo e de uso e manejo. Neste estudo, quanto às práticas conservacionistas $(\mathrm{P})$, adotou-se o coeficiente 1 (plantio morro abaixo), de acordo com Beskow et al. (2009), pois não foi identificada na área nenhuma prática de controle a erosão.

Tabela 2 - Classes mapeadas para uso do solo, áreas de ocorrência, valores do fator $\mathrm{C}$ e correspondentes referências. Fonte: Elaborado pelo autor.

\begin{tabular}{|c|c|c|c|c|}
\hline Uso do solo & $\begin{array}{l}\text { Area } \\
\left(\mathrm{km}^{2}\right)\end{array}$ & $(\%)$ & Fator C & Referéncia \\
\hline Campo assoc, à umidade. & 4,50 & 5,79 & 0,0004 & Oliveirs et al. (2007); \\
\hline Campo de altitude & 16.40 & 21.08 & 0,0194 & Costa ef aL (2005). \\
\hline Área antropizada & 0,19 & 0,25 & 0,0000 & - \\
\hline Cultura & 9,28 & 11,92 & 0,2900 & Ruhoff et al. 2006 \\
\hline Massas d'água & 5,07 & 6.51 & 0,0000 & - \\
\hline Mata Nativa & 0,94 & 1.21 & 0,0080 & Martins et al. (2010) \\
\hline Mata ripária & 8,97 & 11,53 & 0,0120 & Farinasso et al. (2006) \\
\hline Pastagem & 6.65 & 8,54 & 0,0288 & Costa et aL $(2005)$, \\
\hline Reflorestamento & 24,60 & 31,62 & 0,0120 & Farinasso et al. (2006) \\
\hline Solo exposto & 1,23 & 1.58 & 1,0000 & Costa et al. (2005) \\
\hline Total & 37,8 & 100,00 & - & - \\
\hline
\end{tabular}

\section{RESUlTADOS E DISCUSSÃO}

Na Figura 4 (a, b, c e d) apresentam-se as espacializações dos fatores da RUSLE na SBRC. Os valores encontrados para erosividade da chuva (Figura 4a) situaram-se entre $8.983,90$ e $10.634,80$ (MJ.ha ${ }^{-1} \cdot \mathrm{mm}^{-1}$.ano ${ }^{-1}$ ) correspondendo, de acordo com classificação proposta por Mello et al., (2007), a classe de 
"alta" erosividade da chuva. Outra classificação proposta enquadraria os valores aqui estimados como erosividade "muito forte", de acordo com Carvalho (2008).

Observa-se ainda, o gradiente negativo dos valores, a partir dos divisores de agua no sentido dos drenos, sobretudo na cabeceira da SBRC, estando os valores mais elevados de erosividade a noroeste da área, onde ocorrem também as maiores altitudes (Figura 2a), explicitando assim, a elevada e decisiva, influência da altitude nos valores da erosividade.

No caso dos fatores K e C (Figura 4b e 4d) os valores espacializados foram extraídos com base em índices de literatura conforme Tabelas 1 e 2. Na Figura 4c observa-se a distribuição espacial do fator LS, que apresentou uma média de 4,38 sendo os valores mais altos encontrados nas áreas mais declivosas da SBRC, (Figura 2b). A análise espacial do fator LS é de grande importância, pois esse parâmetro influencia diretamente na velocidade do escoamento superficial (energia efetiva do fluxo de água), considerado um dos fatores preponderantes para a ocorrência da erosão. A distribuição espacial e as frequências das classes do fator LS, mostram que $90,05 \%$ da área da SBRC $\left(70 \mathrm{~km}^{2}\right)$ apresenta valores de LS menores do que 10, o qual representa vulnerabilidade moderada à perda de solo, de acordo com Beskow et al. (2009). Em contrapartida, os 9,95\% restantes representam áreas com elevado potencial à erosão (BESKOW et al., 2009). Em termos de área, $6,99 \mathrm{~km}^{2}$ da SBRC apresentaram valores de LS entre $10-20$, sendo que $0,74 \mathrm{~km}^{2}$ apresentaram superiores a 20. Estes valores sinalizam a necessidade de práticas de conservação, a fim de se reduzir o ganho de energia do escoamento devido à topografia (STEINMETZ et al., 2018).

A aplicação da álgebra de mapas sobre os resultados apresentados nas Figuras 4, gerou a distribuição espacial da taxa de perda potencial de solo para a SBRC, cujos valores foram agrupados segundo as classes de vulnerabilidade à erosão, conforme proposição de Beskow et al. (2009), para compor a Figura 5 e a Tabela 3. Ainda na mesma tabela, constam valores de referência, para cada classe de vulnerabilidade (VRCV), utilizados para estimar a taxa média ponderada de perda potencial de solo (TMPPS) nas associações entre Classes de Solo; Declividade e Uso com a vulnerabilidade, que estão contempladas nas Tabelas 4, 5 e 6. Pode-se observar que a classe Ligeira vulnerabilidade com $23,35 \%$ é a de maior frequência na SBRC, seguida pelas classes Moderada e Muito Alta vulnerabilidade, com $18,67 \%$ e com $18,49 \%$ respectivamente. Por outro lado, as áreas com Extremamente Alta vulnerabilidade, representam $8,4 \%$ da SBRC, o que significa que $26,89 \%$ da área da SBRC, proporciona taxas de perdas potencias de solo com valores acima de $25\left(\mathrm{t} \mathrm{ha}^{-1} \mathrm{ano}^{-1}\right)$. Assim, um pouco mais que um quarto da área apresenta taxas de perdas potenciais de solo, com valores muito elevados, influenciando no valor da taxa média ponderada de perda potencial de solo da SBRC que foi de 45,14 ( $\mathrm{t} \mathrm{ha}^{-1}$ $\mathrm{ano}^{-1}$ ), valor este, que a enquadra na classe de Muito Alta vulnerabilidade. Esta estimativa sinaliza também a potencialidade de processo acelerado de degradação do solo, que possivelmente se associa, a áreas com solo descoberto, áreas com cultivos anuais sem práticas de conservação do solo, e também ao efeito negativo de elevadas declividades, conforme se pode observar na Figura 5, e, com mais detalhes nas associações especificas detalhadas nas Tabelas 4, 5 e 6 . Nota-se também, que o valor aqui observado está acima do apresentado por Melo Neto et al. (2017) que estimou uma taxa média ponderada de perda de solo de 10,4 (t ha ${ }^{-1}$ ano $\left.^{-1}\right)$ para a Sub-bacia hidrográfica do Córrego do Gigante, que está contida na SBRC. Esta grande diferença se explicada pela relevante participação espacial das duas classes de maior vulnerabilidade na SBRC em relação à do Córrego do Gigante. 
Tabela 3 - Classes de vulnerabilidade à erosão e quantificação das áreas de ocorrência na SBRC. (*) Segundo Beskow et al. (2009); **VRCV - Valor de referência da classe de vulnerabilidade. Fonte: Elaborado pelo autor.

\begin{tabular}{cccccc}
\hline $\begin{array}{c}(*) \text { Classe de } \\
\text { Vulnerabilidade }\end{array}$ & $\begin{array}{c}\text { Taxas de Perda } \\
\text { Potencial de solo } \\
\left(\mathrm{t} \mathrm{ha}^{-1} \text { ano- }{ }^{-1}\right)\end{array}$ & $\begin{array}{c}* \text { VRCV } \\
\left(\mathrm{t} \mathrm{ha}^{-1} \mathrm{ano}^{-1}\right)\end{array}$ & $\begin{array}{c}\text { Area } \\
\mathrm{km}^{2}\end{array}$ & $(\%)$ \\
\hline Ligeira & $0-2,5$ & 1,25 & 18,12 & 23,35 \\
Ligeirn a Moderada & $2,5-5$ & 3,75 & 8,79 & 11,33 \\
Moderada & $5-10$ & 7,50 & 14,49 & 18,67 \\
Moderada a Alta & $10-15$ & 12,50 & 7,59 & 9,78 \\
Alta & $15-25$ & 20,00 & 7,72 & 9,95 \\
Muito alta & $25-100$ & 62,50 & 14,35 & 18,49 \\
Extremamente alta & $>100$ & 125,00 & 6,52 & 8,40 \\
\hline Total & - & & 77,58 & 100,0 \\
\hline
\end{tabular}

Tabela 4 - Quantificação das áreas de ocorrência da associação entre classes de vulnerabilidade à erosão e classes de solo na SBRC. (*) De acordo com Beskow et al. (2009). ** TMPPS - Taxa média ponderada de perda potencial de solo. Fonte: Elaborado pelo autor.

\begin{tabular}{|c|c|c|c|c|c|c|c|c|}
\hline \multirow{2}{*}{$\begin{array}{l}\text { "Classes de } \\
\text { Vulnerabilidade } \\
\text { (t ha-1 ano }\end{array}$} & \multicolumn{2}{|c|}{$c x$} & \multicolumn{2}{|c|}{ LVA } & \multicolumn{2}{|c|}{ RL. } & \multicolumn{2}{|c|}{ srv } \\
\hline & $\mathrm{kna}^{2}$ & (6) & $\mathbf{k n}^{2}$ & (\%) & $\mathrm{km}^{2}$ & (\%) & $\mathrm{km}^{2}$ & (5) \\
\hline $0.2,5$ & 4,18 & 7,37 & 7,26 & 17,18 & 0,01 & 3,22 & 9.53 & 51.40 \\
\hline $2,5$. & 0.66 & 4.14 & 7,06 & 16,70 & 0.00 & 1,11 & 0.95 & 5.12 \\
\hline $5-10$ & 1,76 & 11,03 & 9.95 & 23,52 & 0,00 & 1,28 & 2,65 & 14,30 \\
\hline $10-15$ & 1,69 & 10,59 & 4,57 & 10,82 & 0,01 & 2,58 & 1,27 & 6,86 \\
\hline $15 \cdot 2$ & 2,82 & 17,67 & 3,68 & 8,71 & 0,02 & 7,27 & 1.19 & 6,41 \\
\hline $25-1$ & 6,74 & 42,26 & 6,06 & 14,34 & 0.12 & 54,42 & 1,32 & 7,13 \\
\hline$>100$ & 1.11 & 6,95 & 3,69 & 8.74 & 0,06 & 30.11 & 1,63 & 8.77 \\
\hline Total & 15,94 & 100,00 & 42,29 & 100,00 & 0,21 & 100,00 & 18,53 & 100,00 \\
\hline${ }^{*}$ TMPPS & & 1.10 & &, 60 & 74,00 & & & 9,5 \\
\hline
\end{tabular}
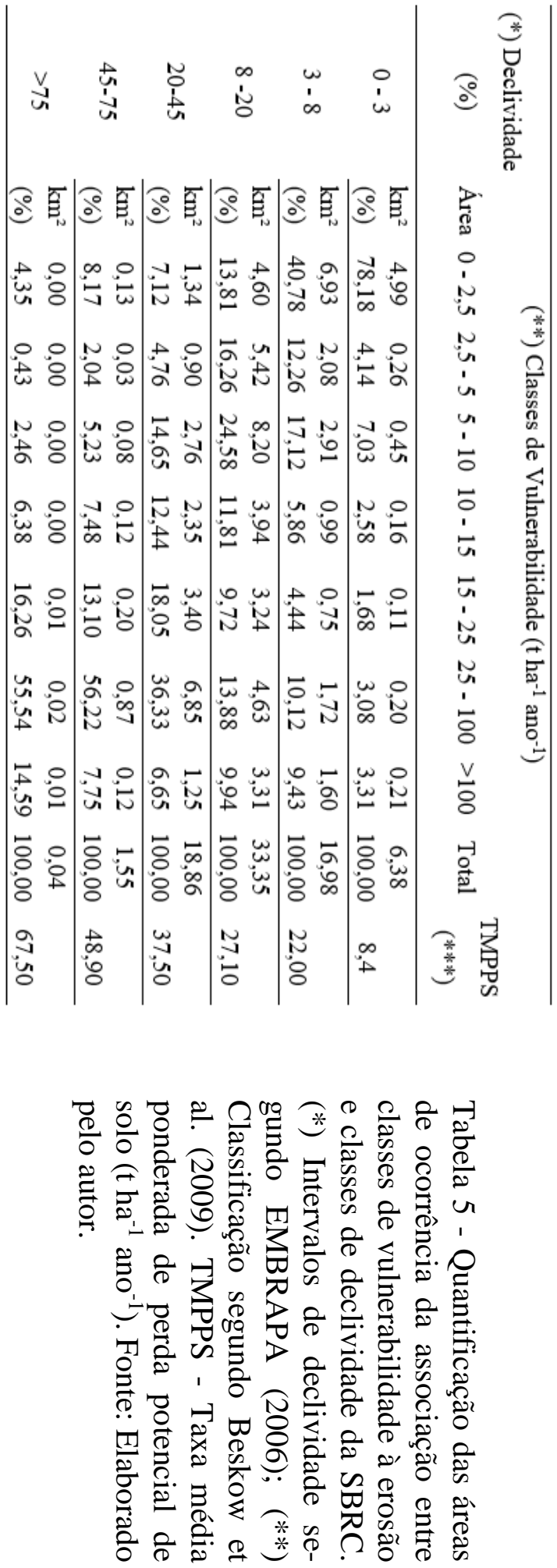


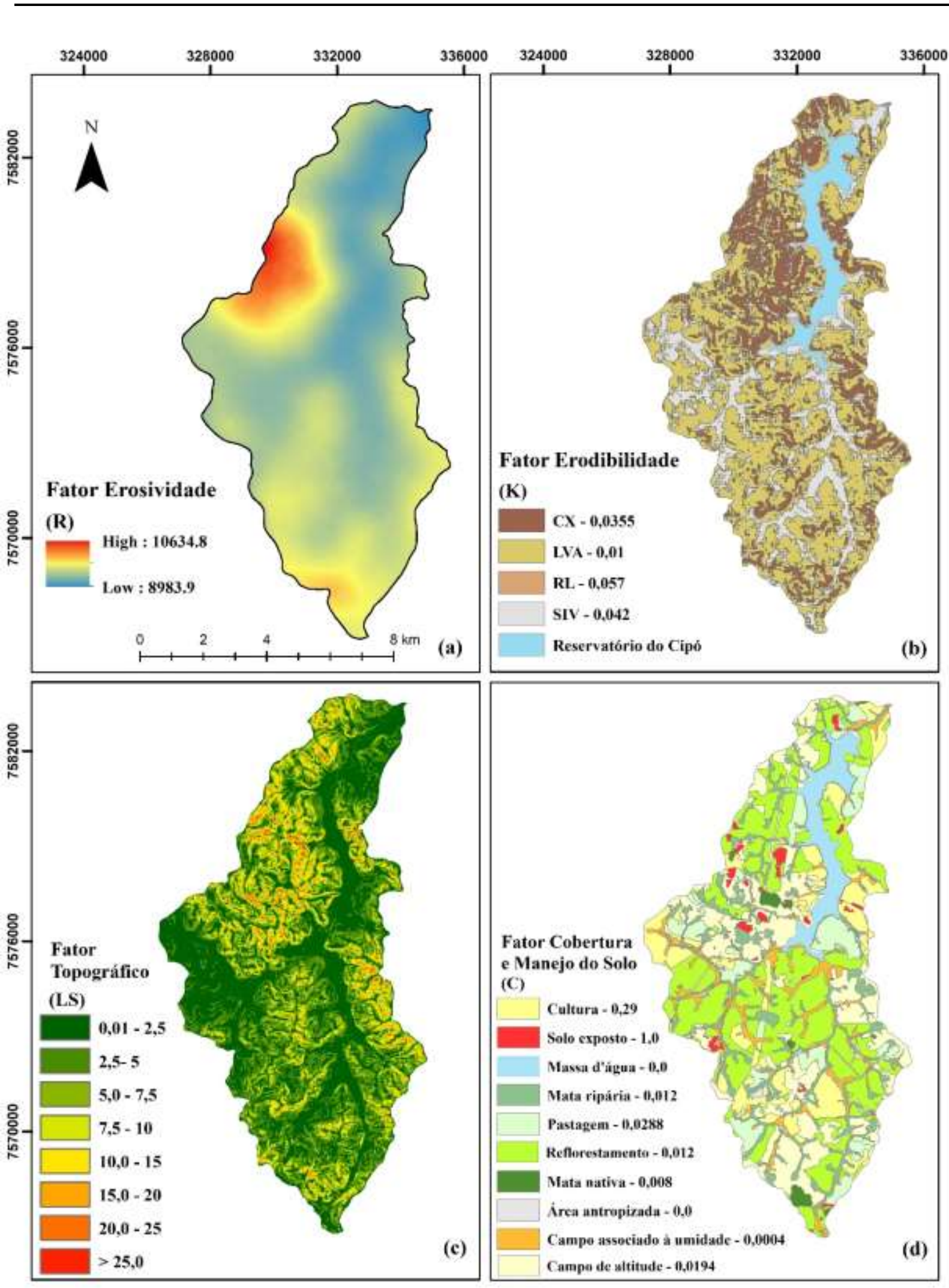

Figura 4 - Fatores da RUSLE espacializados para a Sub-bacia hidrográfica do Ribeirão do Cipó. (a) Fator Erosividade (R), (b) Fator Solo (K), (c) Fator Topográfico (LS) e (d) Fator de Uso de Manejo do Solo (d). Fonte: Elaborado pelo autor. 


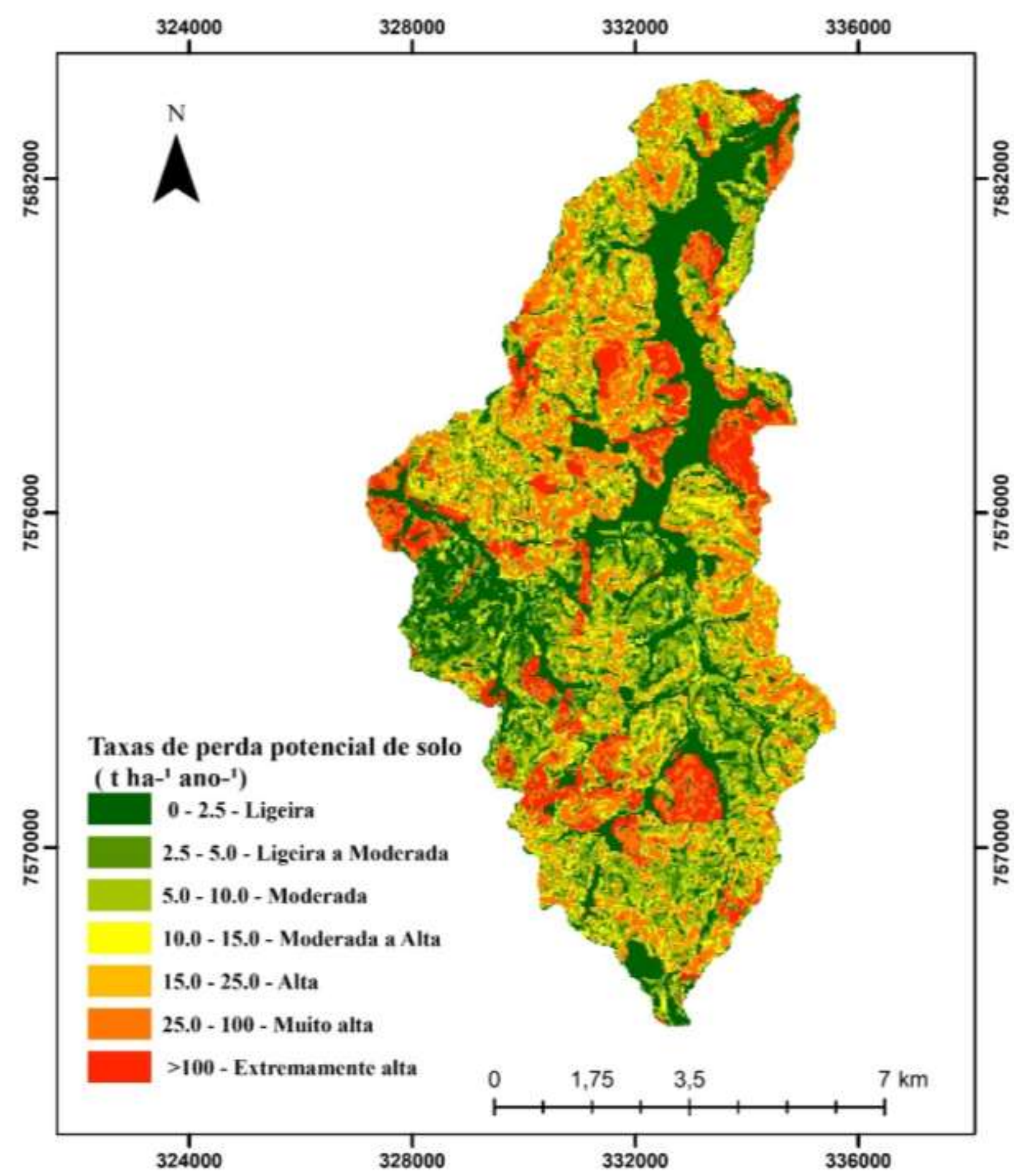

Figura 5 - Espacialização das Taxas de perda potencial de solo $\left(\mathrm{t} \mathrm{ha}^{-1} \mathrm{ano}^{-1}\right)$ na Sub-bacia hidrográfica do Ribeirão do Cipó, conforme classificação proposta por Beskow et al. (2009). Fonte: Elaborado pelo autor. 
Tabela 6 - Quantificação das áreas de ocorrência da associação entre classes de vulnerabilidade à erosão e classes de uso do solo na SBRC. (*) Classes propostas por Beskow et al. (2009). (**) Taxa média ponderada de perda potencial de solo $\left(\mathrm{t} \mathrm{ha}^{-1} \mathrm{ano}^{-1}\right)$. Fonte: Elaborado pelo autor.

\begin{tabular}{|c|c|c|c|c|c|c|c|c|c|c|}
\hline \multicolumn{11}{|c|}{${\text { (*) Classe de Vulnerabilidade ( } \text { ha }^{-1} \text { ano }}^{-1}$ ) } \\
\hline Uso do Solo & Área & $0-2,5$ & $2,5-5$ & $5-10$ & $10-15$ & $15-25$ & $25-100$ & $>100$ & Total & $\begin{array}{c}\text { TMPPS } \\
(* *)\end{array}$ \\
\hline Solo & $\mathrm{km}^{2}$ & 0,06 & 0,04 & 0,06 & 0,03 & 0,04 & 0,08 & 0,90 & 1,22 & \multirow{2}{*}{97,83} \\
\hline Exposto & $(\%)$ & 5,28 & 3,45 & 4,91 & 2,68 & 2,87 & 6,83 & 73,98 & 100,00 & \\
\hline Mata & $\mathrm{km}^{2}$ & 0,73 & 0,02 & 0,02 & 0,01 & 0,02 & 0,04 & 0,02 & 0,87 & \multirow{2}{*}{7,66} \\
\hline Nativa & $(\%)$ & 84,75 & 1,92 & 2,14 & 1,49 & 2,21 & 5,10 & 2,39 & 100,00 & \\
\hline \multirow{2}{*}{ Reflorestamento } & $\mathrm{km}^{2}$ & 4,86 & 5,08 & 6,72 & 2,45 & 2,44 & 2,56 & 0,25 & 24,36 & \multirow{2}{*}{14,20} \\
\hline & $(\%)$ & 19,97 & 20,84 & 27,57 & 10,05 & 10,03 & 10,50 & 1,04 & 100,00 & \\
\hline \multirow{2}{*}{ Pastagem } & $\mathrm{km}^{2}$ & 0,42 & 0,58 & 1,60 & 1,31 & 1,36 & 1,28 & 0,08 & 6,63 & \multirow{2}{*}{22,36} \\
\hline & $(\%)$ & 6,37 & 8,73 & 24,06 & 19,75 & 20,55 & 19,32 & 1,21 & 100,00 & \\
\hline Mata & $\mathrm{km}^{2}$ & 1,86 & 0,83 & 1,89 & 1,14 & 1,16 & 1,83 & 0,26 & 8,97 & \multirow{2}{*}{8.37} \\
\hline Ripária & $(\%)$ & 20,70 & 9,22 & 21,11 & 12,74 & 12,97 & 20,37 & 2,90 & 100,00 & \\
\hline \multirow{2}{*}{ Cultura } & $\mathrm{km}^{2}$ & 0,90 & 0,17 & 0,26 & 0,10 & 0,08 & 3,28 & 4,42 & 9,20 & \multirow{2}{*}{85,18} \\
\hline & $(\%)$ & 9,80 & 1,79 & 2,81 & 1,10 & 0,83 & 35,65 & 48,01 & 100,00 & \\
\hline Campo de & $\mathrm{km}^{2}$ & 0,94 & 1,78 & 3,39 & 2,28 & 2,43 & 4,97 & 0,39 & 16,17 & \multirow{2}{*}{29,05} \\
\hline altitude & $(\%)$ & 5,83 & 11,00 & 20,94 & 14,08 & 15,00 & 30,76 & 2,39 & 100,00 & \\
\hline \multirow{2}{*}{$\begin{array}{l}\text { Campo associado a } \\
\text { umidade }\end{array}$} & $\mathrm{km}^{2}$ & 3,45 & 0,15 & 0,36 & 0,15 & 0,10 & 0,11 & 0,16 & 4,48 & \multirow{2}{*}{8,55} \\
\hline & $(\%)$ & 77,05 & 3,40 & 8,00 & 3,43 & 2,18 & 2,38 & 3,55 & 100,00 & \\
\hline \multirow{2}{*}{$\begin{array}{c}\text { Área } \\
\text { antropizada }\end{array}$} & $\mathrm{km}^{2}$ & 0,15 & 0,01 & 0,01 & 0,00 & 0,00 & 0,01 & 0,01 & 0,19 & \multirow{2}{*}{11,45} \\
\hline & $(\%)$ & 76,86 & 5,63 & 6,49 & 0,87 & 2,59 & 4,52 & 3,05 & 100,00 & \\
\hline
\end{tabular}

A Tabela 4 apresenta a associação da perda potencial de solo em relação às classes de solo presentes na Sub-bacia. Em termos de área, o Latossolo Vermelho Amarelo (LVA) é a classe de solo com maior ocorrência, seguido de Cambissolo Háplico (CX), Solos indiscriminados de Várzea (SIV) e por último o Neossolo Litólico (RL). Quanto às classes de vulnerabilidade, o Cambissolo agrega aproximadamente $50 \%$ de sua classificação entre as classes "Muito Alta e Extremamente Alta". Para o Latossolo, suas maiores porcentagens de área foram encaixadas nas classes entre "Ligeira e Moderada". Já o Neossolo, aproximadamente $85 \%$ de sua área, classifica-se nos intervalos de vulnerabilidade "Muito Alta e Extremamente Alta". Por fim, nas classes de solos SIV mais da metade da área a eles associada situa-se no intervalo de ligeira vulnerabilidade. Em termos de taxa média ponderada de perda potencial de solo (TMPPS), observase na Tabela 4 que o Neossolo Litólico apresenta o maior valor para a TMPPS, fato ligado à maior ocorrência de suas áreas, nas classes de maior vulnerabilidade, com $0,18 \mathrm{~km}^{2}$. Ainda em relação a TMPPS, o Latossolo Vermelho-Amarelo apresentou um valor correspondente a $62,23 \%$ do estimado para o Cambissolo Háplico comportamento este também verificado por Ferreira (2019).

A Tabela 5 exibe a interação entre as taxas potenciais de perdas de solo e as classes de declividade na SBRC. Percebe-se que nos dois primeiros intervalos de declividade (Plano e Suavemente Ondulado) apresentou 
valores até $5 \mathrm{t} \mathrm{ha}^{-1} \mathrm{ano}^{-1}$, enquadrando-se assim como vulnerabilidade "Ligeira a Moderada". As classes de declividade "Ondulado e Forte Ondulado" têm ocorrência de 67,66\% na Sub-bacia, prevalecendo a primeira no intervalo de "Moderada" vulnerabilidade com $24,58 \%$ da área, e a segunda com 36,33\% da área no intervalo de "Muito Alta" vulnerabilidade. Destaca-se ainda que, para os últimos três intervalos de declividade propostos por Embrapa (2006), apesar de menores áreas, estes encontram-se nas classes "Muito Alta e Extremamente Alta" vulnerabilidade. Ressalta-se também, que a TMPPS mostrada para as interações da Tabela 5, apresentou valores de 8,4 ( $\left.\mathrm{t} \mathrm{ha}^{-1} \mathrm{ano}^{-1}\right)$ até $67,5\left(\mathrm{t} \mathrm{ha}^{-1} \mathrm{ano}^{-1}\right)$ de forma crescente com as classes de declividade.

As perdas de solo potenciais em relação a cada uso e cobertura do solo na SBRC estão apresentadas na Tabela 6. Observou-se no mapeamento e também nas visitas a campo, a predominância de Reflorestamento na área de estudo. Considerada a principal atividade econômica na SBRC, apresentou $24,36 \mathrm{~km}^{2}$ se encaixando com as maiores porcentagens nas classes entre "Ligeira e Moderada" vulnerabilidade. A segunda maior área da SBRC em termos de uso e cobertura do solo, está associada aos Campos de altitude com 16,17 km², apresentando maiores porcentagens de área distribuída na classe de vulnerabilidade "Muito Alta". Para os campos associados a umidade, áreas de correlação com a rede de drenagem onde os solos apresentam hidromorfismo e grande presença de material orgânico, 77,05\% de sua área está na classe de Ligeira vulnerabilidade. As Matas Ripárias, com áreas de aproximadamente $8,97 \mathrm{~km}^{2}$, apresentaram maior concentração de área nas classes "Ligeira e Muito Alta" respectivamente, o que acontece também para as áreas de Pastagem, porém com menor ocorrências nas duas primeiras classes (Ligeira e Ligeira a Moderada). As áreas de Mata Nativa apresentaram 84,75 $\%$ de ocorrência na classe de "Ligeira" vulnerabilidade, como esperado a partir da presença de cobertura vegetal. Nas áreas associadas à cultura (geralmente milho e batata) os intervalos estão classificados como "Muito Alta e Extremamente Alta" vulnerabilidade, fator este que pode estar associado com a exposição indireta do solo, como também à falta de práticas conservacionistas na SBRC. Nas áreas de Solo Exposto, observa-se que apesar da área não ser tão elevada como das outras classes de uso, sua maior ocorrência $(73,98 \%)$ está na classe "Extremamente Alta", situação esperada pela susceptibilidade de um solo exposto à erosão hídrica. Os maiores valores para TMPPS foram estimados para solo exposto de 97,83 (t ha ${ }^{-1}$ ano $^{-1}$ ) e Cultura com 85,18 (t ha ${ }^{1}$ ano $\left.^{-1}\right)$, apesar da baixa abrangência em área das classes, como já citado. Em seguida, valores de 22,36 ( $\mathrm{t} \mathrm{ha}^{-1} \mathrm{ano}^{-1}$ ) e 29,05 ( $\left.\mathrm{t} \mathrm{ha}^{-1} \mathrm{ano}^{-1}\right)$ foram estimados para Pastagem e Campo de Altitude respectivamente. Para os usos de Mata Nativa, Mata Ripária e Campo associado à umidade apresentaram menores valores, sendo 7,66; 8,37 e 8,55 ( $\left.\mathrm{t} \mathrm{ha}^{-1} \mathrm{ano}^{-1}\right)$ respectivamente. É importante destacar que, apesar dos valores serem menores dentro dos estimados nas classes, ambos se encaixam no intervalo de "Moderada" erosividade.

\section{CONSIDERAÇÕES FINAIS}

A região da SBRC é caracterizada por elevada erosividade das chuvas, o que potencializa elevados índices de perdas de solo.

A aplicação do modelo RUSLE em associação com o SIG permitiu evidenciar as vulnerabilidades dos solos à erosão hídrica e explicitar via as associações com os componentes do modelo os fatores com maior relevância e a sinalização de aspectos que merecem maior atenção em termos de conservação dos solos.

Em relação a erosividade da chuva, o modelo utilizado evidenciou a participação relevante da altitude na sua estimativa, fato devido ao porte da SBRC. A erodibilidade do solo, fator que embora seja bastante estudado, apresenta grande variação de resultados apre- 
sentados na literatura, no presente estudo evidenciou a menor vulnerabilidade do LVA em relação ao CX, e este em relação ao RL.

Quanto ao relevo, os resultados mostram consistência, onde os maiores potenciais de perdas de solo se associam às áreas com maior declividade.

O uso do solo com mata nativa e reflorestamento concorreu positivamente para a redução da vulnerabilidade à erosão hídrica, e, por outro lado, os cultivos anuais e as áreas de solo descoberto e minerado, sem práticas conservacionistas, elevaram significativamente a vulnerabilidade e consequentemente a taxa de perda potencial de solo, com média ponderada estimada de 45,14 (t ha ${ }^{-1}$ ano $\left.^{-1}\right)$ para a SBRC, situando-a na classe de Muito Alta Vulnerabilidade à erosividade das chuvas. Estes resultados indicam a necessidade de implementação de práticas de conservação do solo para se atenuar as perdas.

Por fim, destaca-se a relevância do SIG para permitir não só visualizar a distribuição espacial das taxas potenciais de perda de solo, mas sobretudo das associações, que demonstram os aspectos mais vulneráveis, e, por consequência a identificação das práticas a serem planejadas e implementadas para a redução da erosão hídricas dos solos.

\section{Agradecimentos}

Os autores agradecem o Conselho Nacional de Desenvolvimento Científico e Tecnológico (CNPq), a Fundação de Amparo à Pesquisa do Estado de Minas Gerais (FAPEMIG) e a Coordenação de Aperfeiçoamento de Pessoal de Nível Superior (CAPES).

\section{REFERÊNCIAS}

ARAÚJO FILHO MC, MENESES, PR, SANO, EE. Sistema de classificação uso da terra com base na análise de imagens de satélite. Rev. Brasileira de Cartografia, Brasília, v. 2, n. 59, p. 171-179, 2007.
BESKOW S; MELLO CR, NORTON LD. Soil erosion prediction in the Grande river basin, Brazil using distributed modeling. Catena, v. 79, n. 1, p. 49-59, 2009.

CARVALHO, NO. Hidrossedimentologia Prática. Editora Interciência, v.2, 599p, 2008.

COSTA TCC, LUMBRERAS JF, ZARONI MJ, NAIME UJ, GUIMARÃES SP, UZÊDA MC. Estimativas de perdas de solo para microbacias hidrográficas do estado do Rio de Janeiro. Boletim de Pesquisa e Desenvolvimento. Rio de Janeiro. v. 78, Embrapa Solos, 2005.

CUIABANO MN, NEVES SMAS, NUNES MCM, SERAFIM ME, NEVES RJ. Vulnerabilidade Ambiental à erosão hídrica na SubBacia Do Córrego Do Guanabara/ Reserva Do Cabaçal - MT, Brasil. Geociências, São Paulo, v. 36, n. 1, p. 138-153, 2017

DMAE (Departamento Municipal de Água e Esgoto de Poços de Caldas). - Plano diretor de abastecimento de água do município de Poços de Caldas - MG. Relatório geral - texto. VOL 1/4. Poços de Caldas, 2013.

EFTHIMIOU N, LYKOUDI E.; PANAGOULIA D, KARAVITIS C. Assessment of soil susceptibility to erosion using the EPM and RUSLE Models: the case of Venetikos River Catchment. Global NEST Journal, Athens, v. 18, n. 1, p. 164-179, 2016.

EMBRAPA, Centro de Pesquisas de Solos. Sistema brasileiro de classificação de solos. 669 - Brasília: Embrapa Produção de Informação; Rio de Janeiro: Embrapa Solos, 2 270 ed. 2006.

FARINASSO M, CARVALHO JÚNIOR AO, GUIMARÃES RF, GOMES, RAT. RAMOS VM. Avaliação qualitativa do potencial de erosão laminar em grandes áreas por meio da EUPS - Equação Universal de Perdas de Solos utilizando novas metodologias em SIG 
para os cálculos dos seus fatores na região do Alto Parnaíba -PI-MA. Revista Brasileira de morfologia, v. 7, n. 2, p. 73-85, 2006.

FERREIRA AM. Modelagem da erosão hídrica dos solos na bacia hidrográfica do Córrego do Gigante - Poços de Caldas MG. 2019. 133p. Dissertação (Mestrado em Recursos Hídricos e meio Ambiente. UNIFAL, Poços de Caldas, 2019.

GASHAW T, TULU T, ARGAW M (2017) Erosion risk assessment for prioritization of conservation measures in Geleda watershed, Blue Nile basin, Ethiopia. Environ Syst Res v. 6, n.1, p.1-14, 2017.

GIANINETTO M, AIELLO M, POLINELLI F, FRASSY F, RULLI MC, RAVAZZANI G, BOCCHIOLA D, CHIARELLI DD, SONCINI A, Vezzoli R. D-RUSLE: a dynamic model to estimate potential soil erosion with satellite time series in the Italian Alps, European Journal of Remote Sensing, v. 52, n.4, p.34-53, 2019.

MARTINS SG, SILVA MLN, AVANZI, SG, CURI N, FONSECA S. Fator cobertura e manejo do solo e perdas de solo e água em cultivo de eucalipto e em Mata Atlântica nos Tabuleiros Costeiros do Estado do Espírito Santo. Sci. For. v. 38, p.517-526, 2010.

MCCOOL DK, FOSTER GR, MUTCHLER, CK, MEYER LD. Revised slope length factor for the universal soil loss equation. Transactions of the American Society of Agricultural Engineers. v. 32, p.1571-1576, 1989.

MELLO CR, SÁ MAC, CURI N, MELLO JM, VIOLA MR, SILVA AM. Erosividade mensal e anual da chuva no Estado de Minas Gerais. Pesquisa Agropecuária Brasileira, v.42, n.4, p.537-545, 2007.
MELLO, CR, VIOLA MR, BESKOW S, NORTON LD. Multivariate models for annual rainfall erosivity in Brazil. Geoderma. v. 202-203, p. 88-102, 2013.

MELLO CR., VIOLA MR., OWENS PR, MELLO JM, BESKOW, S. Interpolation methods for improving the RUSLE R-factor mapping in Brazil. Journal of Soil and Water Conservation, v. 70, n.3, p.182-197. 2015.

MELO NETO JO, SILVA AM, FERREIRA, AM. MENEZES PHBJ, GUIMARAES DV. Vulnerabilidade dos solos à erosão em bacia hidrográfica minerada no sul de Minas Gerais. In: Anais do II Congresso Internacional de Hidrossedimentologia, 2017, Foz do Iguaçu. Rio de Janeiro: Editora Interciência, 2017. v. 1.

MORAES, FT. Zoneamento geoambiental do planalto de Poços de Caldas MG/SP a partir da análise fisiográfica e pedoestratigráfica. 2007, 173p. Tese de Doutorado Instituto de Geociências e Ciências Exatas, Universidade Estadual Paulista, Rio Claro, 2007.

OLIVEIRA, AMM, PINTO, SAF, LOMBARDI NETO F. Caracterização de indicadores da erosão do solo em bacias hidrográficas com o suporte de geotecnologias e modelo preditivo. Est. Geogr., v.5, p.63-86, 2007.

OLIVEIRA VA, MELLO CR, DURÃES, MF, SILVA AM. Soil erosion vulnerability in the Verde River Basin, Southern Minas Gerais. Ciência \& Agrotecnologia, v. 38, n. 3, p. 262-269, 2014.

PRADHAN B, CHAUDHARI A, JAGARLAPUDI A, BUCHROITHNER MF. Soil erosion assessment and its correlation with landslide events using remote sensing data and GIS: a case study at Penang Island, Malaysia. Environmental Monitoring and Assessment. v.184, n.2, p.715-27, 2012. 
PRASANNAKUMAR V, VIJITH H, ABINOD S. GEETHA N. Estimation of soil erosion risk within a small mountainous subwatershed in Kerala, India, using Revised Universal Soil Loss Equation (RUSLE) and geo-information technology. Geoscience Frontiers, v.3, n.2, p. 209-215, 2012.

RENARD KG, FOSTER G R, WEESIES G A, PORTER PJ. RUSLE: revised universal soil loss equation. J. Soil and Water Conservation, v. 46, n. 1, p. 30-33, 1997.

RODRIGUES, JAM, MELLO, CR, VIOLA, MR, RODRIGUES, MC. Estimativa da vulnerabilidade dos solos à erosão hídrica na bacia hidrográfica do Rio Cervo - MG. Geociências, São Paulo, v. 36, n. 3, p.531-542, 2017.

RUHOFF AL, SOUZA BSP, GIOTTO E, PEREIRA RS. Avaliação dos processos erosivos através da equação universal de perda de solos, implementada com algoritmos em LEGAL. Geomática, v. 1, n. 1, p. 12-22, 2006.

SILVA AM, SILVA MLN, CURI N, AVANZI JC, FERREIRA MM. Erosividade da chuva e erodibilidade de Cambissolo e Latossolo na região de Lavras, Sul de Minas Gerais. Revista Brasileira de Ciência do Solo, v, 33, p.1811-1820, 2007.

SILVA MLN, CURI N, LIMA JM, FERREIRA MM. Avaliação de métodos indiretos de determinação da erodibilidade de latossolos brasileiros. Pesquisa Agropecuária Brasileira, Brasília, v.35, n.6, p.1207-1220, 2000.

SILVA VC. Estimativa da erosão atual da bacia do Rio Paracatu (MG/GO/DF). Revista
Pesquisa Agropecuária Tropical. Goiânia: UFG, v. 3, n. 34, p.147-159, 2004.

SILVA AM, SILVA MLN, CURI N, AVANZI JC, FERREIRA MM. Erosividade da chuva e erodibilidade de Cambissolo e Latossolo na região de Lavras, sul de Minas Gerais. Revista Brasileira de Ciência do Solo, v.33, n.6, p.1811-1820, 2009.

STEINMETZ AA, CASSALHO F, CALDEIRA TL, OLIVEIRA, VA, BESKOW SL, TIMM, LC. Assessment of soil loss vulnerability in data-scarce watersheds in southern Brazil. Ciência e Agrotecnologia, v. 4, n,6, p. 575-587, 2018.

Universidade Federal de Viçosa (UFV); Fundação Centro Tecnológico de Minas Gerais (CETEC-MG); Universidade Federal de Lavras (UFLA); Fundação Estadual do Meio Ambiente (FEAM). Mapa de Solos Do Estado de Minas Gerais: legenda expandida. Belo Horizonte: Fundação Estadual do Meio Ambiente, 2010, 49.p.

WISCHMEIER WH, SMITH DD. Predicting rainfall erosion losses: a guide to conservation planning. Agriculture Handbook, 537, Washington. USDA, p. 58, 1978.

ZHANG H, YANG Q, LI R, LIU Q, MOORE, D, HE P, RITSEMA CJ, GEISSEN V. Extension of a GIS procedure for calculating the RUSLE equation LS factor. Computers \& Geosciences. v. 52, p.177-188, 2013.

Submissão: 27/09/2019.

Aceito: 31/05/2020. 